\title{
HISTORIA, EPISTEMOLOGÍA Y DIDÁCTICA DE LAS CIENCIAS: UNAS RELACIONES NECESARIAS
}

\author{
History, epistemology and didactics of science: \\ some necessary relationships
}

\author{
Adriana Patricia Gallego Torres ${ }^{1}$ \\ Romulo Gallego Badillo
}

Resumo: En este artículo se establecen relaciones entre historia, epistemología y didáctica de las ciencias de la naturaleza. De cada una de estas categoría se presenta, además, un análisis de cómo son tratadas en la práctica habitual de algunos sistemas educativos, a la vez que se hacen propuestas para una posible superación. Se propende por hacer objeto de trabajo en el aula cada modelo científico, a partir de una caracterización de las razones que llevaron a la comunidad de especialistas a formularlo y aceptarlo. El objetivo es el de propiciar una reflexión fundamentada para una investigación didáctica, en la que esas relaciones superen habituales miradas aisladas.

Palavras-clave: Historia. Epistemología. Didáctica. Triangulación de relaciones entre estas categorías.

\begin{abstract}
In this article, the authors present relevant relationships between history, epistemology and didactics of natural sciences. Each of these categories is analyzed based on the real practice of some educational systems and some proposals are outlined in order to improve them. Each one of the scientific models should be the focus of classroom work, taking into account the characterization of the foundations which have led the scientific community to state and accept them. The main objective is to start a reflection based on didactic research in which the established relationships overcome different isolated points of view.
\end{abstract}

Key words: History. Epistemology. Didactics. Triangulation of established relationships between categories.

${ }^{1}$ Doctora en Didactica de las ciencias; profesora, Planta del Doctorado Insterinstitucional, Universidad Distrital Francisco José de Caldas. Bogotá, Colômbia. <adpgallegot@udistrital.edu.co>

${ }^{2} \mathrm{Mg}$ en Educacion; profesor titular, Universidad Pedagogica Nacional de Colombia. Bogotá, Colômbia.

<rgallego@uni.pedagogica.edu.co>

${ }^{1}$ Calle $13, n^{\circ} 8-39$

Barrio La Alegría, Chía

Cundinamarca, Colombia 
Gallego Torres, A. P.; Gallego Badillo, R.

\section{Introducción}

Ha sido habitual mostrar las ciencias de la naturaleza como obra de personajes geniales e independientes de sus respectivos contextos sociales, culturales, políticos y económicos (GALLEGO TORRES, 2002); es decir, los responsables de la formulación de los distintos modelos científicos son presentados en los sistemas educativos, sin mencionar siquiera las problemáticas que en su momento enfrentaba la comunidad de especialistas en la disciplina científica correspondiente; disciplina que tampoco es contextualizadas cultural, social, política y económicamente. De esta manera la historia de cada una de las ciencias de la naturaleza, no emerge como necesaria para comprender los procesos internos y externos que condujeron a las formulaciones, aceptaciones, transformaciones y cambio de los modelos. Cuando de alguna manera se alude a la historia, esta por lo general se reduce a una narración lineal y acumulativa de descubrimientos, acompañada por una sinopsis biográfica de esos genios, en la que se destacan los descubrimientos que llevaron a cabo. En este orden de ideas esa alusión está referida a un complemento, a una especie de agregado cultural, que poco o nada tiene que ver con la pregunta de cómo y por qué se formuló y admitió un modelo dado, entre todos los otros que para la misma época competían, con miras a ser aceptados como explicaciones válidas de la problemática epistemológica de ese entonces.

Los exámenes en torno a la ausencia de una historia de los modelos científicos que se hacen objeto de trabajo en el aula, parecen demostrar que la aproximación epistemológica dominante es la empiropositivista, dentro de una mirada que los presenta limitados a un resumen meramente tecnicista, esto es, esquematizados en definiciones y en fórmulas matemáticas (cuando es necesario), que han de ser aplicadas para la resolución de ejercicios del tipo de lápiz y papel, además de los ejercicios contenidos en las páginas finales de los capítulos de los textos de enseñanza.

Además, las prácticas de laboratorio se inscriben en la misma esquematización, puesto que obedecen al seguimiento de unas guías previamente seleccionadas por el profesor; guías que, por lo general, no dejan de ser un recetario lineal de procedimientos, así se justifiquen con el argumento de que los estudiantes han de ser entrenados en las técnicas ya probados y en el manejo eficiente de los instrumentos y técnicas estandarizadas.

Puesto que el objetivo es la transmisión verbal y la repetición memorística de contenidos curriculares, en el interior de esa mirada tecnicista y desde la aproximación empiropositivista, la historia de las ciencias no se hace aparecer como indispensable, para que los estudiantes se acerquen a una comprensión racional de las razones por las cuales se formularon y aceptaron los modelos y el papel que para cada uno de esos modelos, cumplieron los resultados experimentales y la necesidad de un desarrollo tecnológico consignado en los instrumentos. En otras palabras y como lo demuestran investigaciones didácticas, en la praxis educativa no se trabaja con modelos, ni se analiza el papel cumplido por los experimentos que les dieron apoyo empírico.

Por otro lado, es preciso señalar que desde la aproximación epistemológica empiropositivista dominante, cada una de las ciencias existe en la naturaleza, por lo que el trabajo es descubrirlas mediante el empleo del método científico, dejando de lado las críticas que sobre 
Historia, epistemología y didáctica...

este modelo han sido realizadas por especialistas (ZAHAR, 1980). Este dominio y praxis educativa instauran, además de la idea de que esas ciencias o la reducción que de ellas se hace, el convencimiento de que se encuentran consignadas en los textos de enseñanza, de tal manera que ellas son tal cual como esos textos las presentan a los estudiantes. Agréguese a lo anterior, que parece ser usual que los profesores de ciencias sean formados en la ciencia en la que se desempeñarán, y justamente dentro de las versiones que imponen dichos textos (GALLEGO BADILLO et al., 2004; GALLEGO BADILLO et al., 2004).

En lo tocante a la didáctica de las ciencias de la naturaleza, los estudios realizados conducen a afirmar que sigue siendo concebida, por un lado, como la parte metódica o instrumental de la pedagogía, considerándose esta como la ciencia de la educación. Se tiene entonces un problema epistemológico, por cuanto el proceder en las instituciones educativas y en el aula, no se deriva conceptual y metodológicamente de los presupuestos teóricos de dicha ciencia. Por otro lado, esa didáctica, así reducida, se queda en el contexto de una práctica meramente artesanal. En este orden de ideas, cualquier análisis que se haga de la historia de las ciencias y con las aproximaciones epistemológicas que fundamentan esa reducción, tampoco se hace aparecer como necesario. Su consecuencia inmediata es que el profesor de ciencias es catalogado como un simple operario del sistema educativo, y toda la información recolectada, parece hablar a favor de que en los programas de formación inicial y continua de profesores de ciencias, los currículos y el trabajo de los formadores de formadores están encaminados a que esos profesores de ciencias se desempeñen profesionalmente como simples operarios, tal cual como el sistema en este caso, espera.

Algunas de las investigaciones adelantadas por los responsables del presente artículo, han contrastado lo ya aceptado por la comunidad de didactas de las ciencias, en cuanto que aquellos que por una razón u otra deciden formarse inicialmente como profesores de ciencias, de que ya han elaborado una concepción de ciencia, una imagen de científico y de actividad científica y de lo que significa ser profesor de ciencias. Concepción e imagen elaborada de manera no reflexionada histórico-epistemológica ni didácticamente, puesto que son producto de obedecería a sus experiencias escolares anteriores, y de sus empatías con uno, dos o más profesores de ciencias que tuvieron en la educación secundaria. Al respecto, las investigaciones en la nueva didáctica de las ciencias, han concluido que esa formación ha de ser replanteada en términos de un cambio epistemológico, didáctico y pedagógico (PORLÁN, 1989).

El otro problema que cobra importancia epistemológica, histórica y didáctica, es el referido a aquellos profesionales formados en programas profesionales de ciencias, para quienes por una u otra razón deciden desempeñarse como docentes. Para ellos, los interrogantes anteriores siguen siendo válidos ¿Cuál es la versión de ciencia que han elaborado? ¿Cómo conciben la enseñaza de las ciencias? ¿Qué imagen de profesor de ciencia repiten? ¿Desde dónde sostienen la ciencia que reclaman enseñar? ¿Desde los textos de enseñanza y las prácticas repetitivas de laboratorio en los que se centró su enseñanza? ¿Sus profesores basaron las exigencias con base en las publicaciones admitidas en revistas especializadas? ¿De esta manera, cómo ubicar científicamente las exigencias de profesores no reconocidos por la comunidad de especialistas? ¿En qué revistas de la comunidad han admitido artículos derivados de investigaciones? ¿Quién cita como apoyo los resultados de las investigaciones realizadas? 


\section{La historia de las ciencias como problema epistemológico y didáctico}

Los estudiosos de esta problemática saben que la concepción actual sobre la importancia de la historia de las ciencias, se inició con las propuestas de Thomas Kuhn (1972), a partir de las cuales se instauró el convencimiento de que no era posible una aproximación epistemológica acerca de qué eran las ciencias de la naturaleza y cómo se habían desarrollado y construido, por fuera de una revisión histórica documentada. En esta propuesta Kuhn, enseñó que recurrir a los documentos originales en los que la comunidad científica había expresado sus modelos, era la fuente indispensable de una propuesta histórico-epistemológica. Desde una interpretación didáctica, Kuhn (1972), puntualizó que los profesores de ciencias enseñaban historia, es decir, paradigmas ya elaborados o superados, sin seguir la lógica de la investigación histórica.

Lakatos (1983) introdujo el planteamiento de que con respecto a la historia de las ciencias, era necesario plantearla en términos de reconstrucción; una reconstrucción que estaba marcada por la opción epistemológica de quien se ocupaba de demostrar, en el presente, cómo se habían producido, sostenido y abandonado cada uno de los programas de investigación científica; programas estos, que como categoría epistemológica, este especialista dio cuenta del desarrollo de las ciencias, teniendo como punto de reflexión lo sucedido en la física de finales del siglo XIX y comienzos del siglo XX. Una libre lectura de lo apuntado por este autor, da pie para pensar que habría diferentes versiones de esa historia, entre las cuales se contaría la habitual ya comentada, esto es, la de la acumulación lineal de descubrimientos llevada a cabo por esos genios, cuyas biografías simplificadas aparecen en algunos textos de enseñanza.

Anótese además, que esa simplificación es eminentemente sexista, ya que está referida al papel protagónico cumplido por hombres, desconociéndose las actuaciones significativas llevadas a cabo por mujeres que se destacaron en ese desarrollo. Así se caiga en casuística, hay que traer como ejemplo, en el caso de la química, la importante labor desempeñada por la esposa de Lavoisier, Marie Anne Pierrette Paulze (1758-1836), en la divulgación de la teoría de la oxidación. Es de anotar también, que esas bibliografías simplificadas se caracterizan por suprimir los rasgos humanos de esos hombres, hasta el punto de que se destacan en grado superlativo sus cualidades como científicos y se borran las "debilidades" o comportamientos que contribuyen a caracterizarlos como pertenecientes a la especie.

Retornando a Lakatos (1983), en su propuesta hace referencia a la necesidad de una historia interna y una historia externa de los programas de investigación, aun cuando estipula que la mejor historia externa es la interna, por cuanto da cuenta de las razones por las cuales cada programa se hizo progresivo, hasta que su dudoso apoyo empírico contribuyó a su abandono definitivo. Hay que entender al respecto, que se está frente a una propuesta epistemológica en la que los análisis culturales, sociales, políticos y económicos ocupan un lugar secundario. Hay la impresión de que el trabajo de producción de saberes científicos es connatural a las sociedades que lo adoptaron, por lo que la pregunta por qué las de los llamados del tercer mundo o en vías de desarrollo no lo hicieron carece de importancia. Al respecto, como se recordará, se afirmó que eso del método científico había que dejárselo a los subdesarrollados, ya que nunca harían ciencia (FEYERABEND, 1984).

Por otro lado, la historia de las ciencias por fuera de su relación dialéctica con los distintos saberes tecnológicos, es incompleta, para no decir que mutilada. De todos es conoci- 
do que dicha relación fue introducida por Galileo, con la creación de una nueva versión de ciencia, de carácter matemático e instrumental; instrumentos que al principio fueron artefactos fabricados por los maestros artesanos, para después ser tecnofactos. Estos tecnofactos surgen de la también introducción de la episteme de las matemáticas en el saber de los artesanos, lo que lo transformo en tecnología. A partir de ese momento histórico las ciencias de la naturaleza y los saberes tecnológicos se han venido desarrollando con apoyos conceptuales y metodológicos mutuos. Sin investigación científica no hay investigación tecnológica y viceversa.

Los autores del presente artículo se atreven a plantear que en la actualidad, las investigaciones en cada una de las ciencias de la naturaleza, se objetivan y se hacen "visibles" para la gente del común a través de los tecnofactos que convertidos en mercancía, se ofrecen para todos en el mercado; planteamiento este que no recurre a la mirada positivista y no histórica, de que las tecnologías son una aplicación del conocimiento científico a los sistemas de producción. Otra forma de hacer "visible" para la mayoría de las personas, los resultados de la actividad de las comunidades científicas, pasa por las intencionalidades de los sistemas educativos y la formación inicial y continua de profesores de ciencias, cuando el objetivo es trabajar con los estudiantes los cambios en las concepciones de mundo y de la práctica científica misma, que trajeron como consecuencia la sustitución de teorías o los cambios paradigmáticos.

De hecho, los estudios realizados por los sociólogos de las ciencias, parecen demostrar que estas se han construido en entornos democráticos, en los que el pensar de una manera distinta es tolerado por la mayoría de los ciudadanos y por quienes principalmente ostentan el poder político. De la misma manera, por una sociedad que ha salido de una etapa de producción artesanal a la industrial y por ende competitiva. Sociedad que ha logrado un consenso mayoritario en el que el pensar y producir conocimiento científico ha tomado distancia y se ha acordado que nada tiene que ver con las creencias religiosas, puesto que se ha comprendido, gracias al trabajo de los didactas, que los científicos no se ocupan ni se han ocupado históricamente, de problemáticas análogas. Se trata de sociedades en las que se ha llevado a cabo una superación crítica de la mirada aristotélica de que los seres humanos conocen por curiosidad y que cualquier idea acerca del funcionamiento del mundo es potencialmente válida y aceptable. En este contexto, la relación ciencia, tecnología e industria no es objeto de discusión.

Dígase entonces, que las ciencias requieren de nichos culturales, sociales, políticos y económicos propicios, en los que el sistema educativo procure instaurar en las futuras generaciones una concepción de ciencia y una imagen de científico (a), acorde con una reconstrucción histórica mucho más próxima a las maneras como cada una de las ciencias de la naturaleza fue desarrollada. Esto significa un no privilegio del denominado método científico y de unas supuestas competencias científicas de carácter empiropositivista, que, además de ser ahistóricas, desconocen cómo las distintas ciencias de la naturaleza han obedecido a todo un esfuerzo colectivo (HODSON, 1985). Stephen Toulmin (1977), sostuvo que las ciencias han sido producto de una genealogía de maestros y discípulos; ello significa que el desarrollo de las ciencias está ligado a los objetivos de los sistemas educativos, a las razones y concepciones históricoepistemológicas y didácticas de los niveles que lo conforman y al desempeño de profesores y profesoras, en consonancia con sus entornos culturales, sociales, políticos y económicos, además del sentido que le confieren a ese desempeño, esto es a su proyecto ético de vida como profesores.

Desde posiciones deductivistas, una historia interna de las ciencias ha de ocuparse de 
las problemáticas que condujeron a la formulación de teorías, de paradigmas o de modelos científicos; de la estructura conceptual y metodológica de cada uno que se admitió como respuesta a esas problemáticas, de los apoyos empíricos que contribuyeron a su admisión por parte de la correspondiente comunidad de especialistas (GALLEGO BADILLO et al., 2004). Ha de examinar aquellos problemas que planteados en el interior de cada estructura, las soluciones respectivas no fueron satisfactorias y condujeron a la posibilidad de teorías (POPPER, 1962; LAKATOS, 1983) sustitutas, cambios paradigmáticos (KUHN, 1972) o de transformación de los modelos (SCHELER, 1926; GIERE, 1990; HANSON, 1977; TOMASI, 1999; DEL RE, 2000; CALDIN, 2002; KRETZENBACHER, 2003) existentes.

Como problema didáctico, se estipula en este artículo, que ha de superar la idea inicial e importante, de que la introducción de la historia de las ciencias en los niveles de educación básica y media (secundaria) contribuye a que los estudiantes se aproximen a una idea fundamentada acerca del carácter de cada una de las ciencias de la naturaleza (MATTEWS, 1998) como actividad humana, adelantada por una comunidad de especialistas, dentro de un contexto cultural, social, político y económico, que puede ser identificado y caracterizado para cada época. Ese ir más allá aboga por la necesidad de formular relaciones necesarias entre la historia de cada ciencia y la nueva didáctica de las ciencias; relaciones que tampoco se circunscriben al también problema de la transposición didáctica (ACEVEDO et al., 2005).

\section{Los fundamentos para una historia de las ciencias}

El problema que se presenta cuando se intenta hacer una reconstrucción histórica de una teoría o modelo científico, es el de la selección de las fuentes que han de fundamentar ese propósito. La decisión ha de optar por el examen de de las revistas especializadas en que fueron publicados esas teoría o modelos, admitidos, desarrollados e, incluso, sustituidos o transformados por la correspondiente comunidad científica. Esta decisión ha de tener claro lo tocante a que hubo libros, unos no estrictamente didácticos y otros con esta intencionalidad, en los que se expusieron teorías y modelos que determinaron nuevas miradas y concepciones acerca de la problemática científica de su época.

En los inicios modernos de cada una de las ciencias experimentales, las nuevas teorías o modelos fueron presentados a la comunidad en libros. Isaac Newton (1642-1727), da a conocer su Principios matemáticos de filosofía natural, en 1687 y su Óptica, en 1704, a partir de las cuales se impone la idea de lo que ha de ser y como se tiene que escribir un conjunto de proposiciones para ser considerado como científico. Robert Boyle (1627-1691), en 1661 pone a circular El químico escéptico y en 1666, Orígenes de las formas y de las cualidades. Lavoisier (17431794), en 1789, da a la luz su Tratado elemental de química, aun cuando había ya publicado muchos de sus trabajos en las Mémoires de l'Académie des Sciences, en el Journal de Physique y en los Annales de Chemie.

Richter (1776-1810), entre 1792 y 1793 da a la publicación los tres tomos, Principios de estequiometría o ciencia de medir los elementos químicos. Dalton (1776-1844) en 1807 da a la luz pública su A new sistem of chemical phylosophy. Cannizzaro (1826-1910) da a conocer su Sunto di un corso di filosofia chimica, en 1858. Jacobus Henricus van't Hoff (1852-1911) da para la edición su Estudios de dinámica química. Meyer (1830-1895), hizo lo mismo con su Teorías de la química 
Historia, epistemología y didáctica...

moderna, en 1864. En 1869, Mendeleief (1834-1907) escribe su Ensayo de un sistema de los elementos fundado en el peso atómico; los anteriores para sólo mencionar algunos de los más relevantes en física y en química.

En biología, Malpighi (1628-1694) en 1671 da a la luz su Anatome plantarum.

Lamarck (1744-1829), en 1800 publica su Recherches sur l'organisation de corps vivants; de 1809 es su Phylosophie zoologique y con fecha de 1815, Histoire naturelle des animaux sans vertèbres. Wallace (1823-1913) dio a la imprenta en 1858 Sobre las tendencias de las variedades a separarse indefinidamente del tipo original. En 1859, Darwin (1809-1882) publica su El origen de las especies; en 1859, La variación de los animales y las plantas por la domesticación. Haeckel (1834-1919), en 1885 publica su Generelle Morphologie der Organismen. En 1858, Virchow (1822-1902), da a conocer Die Cellularpathologie. Por supuesto que una consulta de mejor detalle enlistará un número mayor de libros que fueron claves en el desarrollo de la biología como una de las ciencias de la naturaleza.

Muchos de estos libros, sobre todo los de química, fueron redactados con propósitos didácticos, es decir, con la intencionalidad de introducir a las nuevas generaciones en una mirada sobre esta ciencia, diferente de la habitual y dominante, siendo cada uno de ellos textos científicos. Otra cosa sucederá con los textos escolares propiamente dichos, cuyo origen parece deberse a Comenio (1592-1670), partidario de la propuesta empirista de Francis Bacon (1561-1626). Para Comenio, en su Pampedia, establece que el enseñante no ha de ocuparse de qué enseñar y cómo adelantar esta actividad, ya que son otros los encargados de establecerle dicho horizonte, por lo que el libro de enseñanza tendría que adaptar los conocimientos a esa condición de operario que él le confiere al enseñante.

Destacando como se ha hecho, los libros en que muchos de los científicos líderes de sus grupos de trabajo propusieron a su respectiva comunidad de especialistas las soluciones discutidas y reflexionadas acerca de las problemáticas científicas y epistemológicas de su tiempo, es necesario subrayar y destacar que a partir del siglo XVII y con mayor certeza en el XVIII y así posteriormente, los colectivos de científicos decidieron fundar y apoyar revistas especializadas en las que dan a conocer sus formulaciones teóricas. Tal actitud comunitaria trajo como consecuencia, en primer lugar, el establecimiento de unas normas por parte de los respectivos comités editoriales, y someter a evaluación por parte de especialistas las propuestas procedentes de esos colectivos.

De hecho, se caería en un anecdotario si dichos libros se dejaran en la sola referencia de que fueron escritos por los respectivos científicos. El análisis histórico tiene que establecer comparaciones críticas en las que se destaquen, en primer lugar, los aportes que supervivieron al paso del desarrollo de la actividad científica; segundo, y aquellas propuestas que se señalan hoy como erradas, pero que tuvieron su valor en el contexto de las primeras; y, tercero, el examen de cada uno de los textos de los diferentes autores, con miras a tipificar epistemológicamente, lo primero y lo segundo antes señalados.

En segundo lugar, se instauró también norma, de reconocer el estatuto científico de cada colectivo con base en los artículos publicados en esas revistas especializadas e indexadas; en otras palabras, se es científico porque se han aceptado y publicado en dichas revistas los resultados de sus trabajos de investigación en ese campo de producción de saber científico. Esta situación ha dado pie para que se haga la pregunta ¿en qué revista circula su discurso? Mucho más allá, hay la tendencia a validar en la respectiva comunidad de especialistas el discurso científico que se ha propuesto, siempre y cuando otros colectivos de investigadores, en 
revistas diferentes a aquella en la que se aceptó el artículo, fue evaluado y publicado, hagan referencia crítica a dicho artículo. Parecería que en la actividad de producción de conocimiento científico, los títulos académicos no constituyen garantía de reconocimiento por parte de la comunidad internacional de científicos.

Con miras a poner de presente lo hasta aquí desarrollado, se enlista una relación del nacimiento de las revistas especializadas. La historia de las ciencias muestra que a partir de 1666, con la creación de la Philosophical Transaction, de la Royal Society, los científicos comenzaron a publicar sus teorías o modelos en las revistas especializadas, una práctica que se hizo frecuente a finales del siglo XVIII y que se estableció definitivamente en el siglo XIX; así, en 1869 nació Nature. En cuanto al uso de los nombres de las ciencias actuales, 1797 apareció en Inglaterra una revista no institucional, el Journal of Natural Philosophy, Chemistry and the Arts, en 1794, se funda el no institucional Journal de Physique, de Chimie, et d'bistoire Naturelle, en Francia, En este país, eentre 1809 y 1840 se editó Annales de Physique et de Chemie.

En Alemania, la primera fue el Jornal der PhysiK, en 1790, que sufrió cambios de nombre: en 1799 se denominó Annalen der Physik, en 1819 Annalen der Physik un der physikalischen Chemie y en 1824, Annalen der Phisik und Chemie en 1790, la también no institucional Zeitschrift der Physik. Hay que anotar que en 1832, por cambio en el nombre inicial, cobra vida el Pbilosophical Magazine and Journal of Science, por lo que se es del parecer que es a partir de esta revista que la palabra ciencia comienza su uso generalizado entre las comunidades de especialistas. En Estados Unidos en 1818, se publicó el American Journal of Science. Con la fundación en 1876 de la American Chemical Society, esta publica en 1879 el Journal of the American Chemical Society. En el siglo XX se multiplican las revistas especializadas, con la división de cada una de las ciencias en sus disciplinas.

La dinámica de las revistas especializadas ha impuesto un reconocimiento para cada uno de los miembros de los grupos de investigación que se ocupan de producir conocimiento en las distintas disciplinas de las ciencias de la naturaleza. En este sentido, los correspondientes comités editoriales han establecido unas normas, para admitir en las páginas de las respectivas revistas unas normas que deben seguir quienes aspiran a publicar los resultados de sus trabajos en una de estas revistas. Además, esas revistas cuentan con un equipo de evaluadores de reconocido prestigio comunitario, quienes deciden si la contribución posee la suficiente calidad para ameritar ser publicada. Después vendrán las revisiones y sugerencias para hacer las correcciones. Todo aquel que persigue ser reconocido como científico, se aplicará a reescribir su contribución como lo exigen los evaluadores.

Si bien cada quien tiene el derecho de expresar aquello que piensa sobre una temática científica, lo cierto es que, tan criticable como se quiera, se suele preguntar ¿en qué revista especializada ha sido admitido y circula su discurso? Para insistir, si esto no es demostrable, sin importar cuan interesantes y valiosas sean sus ideas, lo cierto es que no son consideradas como para ser referenciadas. Es más, las exigencias de reconocimiento se han extremado, hasta el punto de que hoy se revisa qué otros investigadores de alto nivel, en revistas diferentes, citan de manera crítica esa contribución, con la que se aspira al reconocimiento como científico. La vieja y ya pasada de moda costumbre de anteponer los títulos de posgrado, doctorado y posdoctorado, por sí solos no constituyen garantía para ese reconocimiento, en un entorno científico competitivo. La norma es la producción.

Las afirmaciones anteriores han de ser tomadas con beneficio de inventario. Hoy se 
Historia, epistemología y didáctica...

sabe que a Newton no le publicaron un artículo acerca de la óptica en el Philosophical Transaction, del cual fue director más tarde y en el que nunca puso a circular un artículo (LEIDLER, 1995); informes de prensa recientes hablan a favor de que hubo una revista especializada que le rechazó un artículo a A. Einstein, a su vez, parece que juró, que nunca enviaría una contribución suya a dicha revista; Hoffmann (1997), Premio Nobel de Química, relata que a él también revistas de su especialidad le rechazaron cuatro publicaciones; el artículo en el Ruelle y Takens (RUELLE, 1993) proponían el concepto de atractor extraño, significativo hoy en la dinámica de los sistemas no lineales (BRIGGS e PEAT, 1990), fue igualmente rechazado por los evaluadores de una revista especializada, hasta el punto de que crearon una propia para darlo a conocer. Se es del convencimiento de que estos hechos son normales dentro de la historia de las ciencias; y, que, sólo los miembros de las comunidades científicas pueden hacer de ellos parte de las anécdotas de sus biografías como productores de saberes.

Puesto que en este artículo se sostiene que la nueva didáctica de las ciencias es hoy una disciplina conceptual y metodológicamente fundamentada, anótese que los investigadores en la nueva didáctica de las ciencias de la naturaleza publican los resultados de sus trabajos en más de una treintena de revistas especializadas, muchas de las cuales son indexadas. Si bien no se relacionan las fechas de nacimiento de cada una de ellas, a continuación se mencionan las más conocidas en el contexto iberoamericano. Señálense por ejemplo, el Journal Science Education, el Intenational Journal of Science Education, Science Teaching, Physic Teaching, Biology Teaching, Journal of Chemical Education, Enseñanza de las Ciencias, Ciência \& Educação, la argentina Revista de Enseñanza de la Física, la mejicana Educación Química y la colombiana Tecné, Episteme y Didaxis. Si el nombre de un grupo de especialistas no aparece en uno de los números de estas revistas, su discurso como didacta o pedagogo de las ciencias carga con la misma calificación.

Hecha las relación anterior, se retoma la pregunta, en su perspectiva didáctica ¿dónde se encuentra cada una de las ciencias de la naturaleza?, ¿'en la naturaleza misma?, ¿en los textos de enseñanza? Las respuestas a estos interrogantes, si se elaboran desde una aproximación empiropositivista, estarán del lado de la idea de que se encuentra en la naturaleza y que el trabajo de los hombres y mujeres de ciencias es descubrirla. Si es con base en una aproximación deductivista, las respuestas afirmarán que se encuentra en las correspondientes revistas especializadas. Para ser más específico, en esas revistas se encuentran las teorías y modelos científicos que conforman cada una de esas ciencias de la naturaleza, además de los resultados de las investigaciones que les confieren apoyo empírico. En lo tocante a los modelos, la revisión de la literatura en la que se discute la caracterización de esos modelos (SCHELER, 1926; GIERE, 1990; HANSON, 1977; LOMBARDI, 1998;TOMASI, 1999; DEL RE, 2000; CALDIN, 2002; KRETZENBACHER, 2003), los diferentes autores concluyen y hay ciertos acuerdo entre ellos, que los modelos no son la naturaleza ni la realidad en sí, ya que son representaciones idealizadas de una porción de esa naturaleza o realidad, que se modeliza para hacerla objeto de estudio y de investigación sistemática.

Los interrogantes formulados son también válidos para indagar sobre ¿̇a qué didáctica se hace referencia?, ¿en qué revistas se encuentran los planteamientos y modelos de la nueva didáctica de las ciencias?, ¿qué significado atribuirles, para una historia de la constitución de esa nueva didáctica: a lo libros sobre didáctica, en los que se considera que es la parte operativa de la pedagogía?, ¿cómo fundamentar a partir de las revistas enlistadas y de otras del mismo nivel, las relaciones entre historia, epistemología y didáctica? 
Gallego Torres, A. P.; Gallego Badillo, R.

\section{E1 triangulo de relaciones}

Lo tratado hasta aquí puede ser sintetizado en un triángulo de relaciones entre la historia $(\mathrm{H})$, la epistemología (E) y la didáctica de las ciencias (D), como se recoge en la siguiente figura:

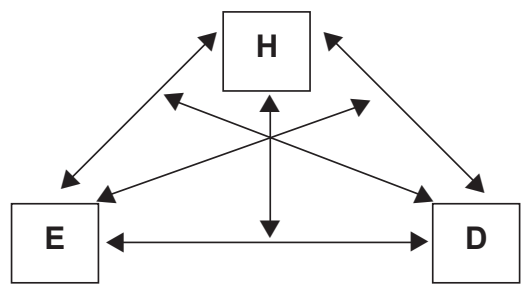

La relación historia $(\mathrm{H})$ y epistemología $(\mathrm{E})$, es la que determina desde que mirada se hace la reconstrucción histórica y, en consecuencia, estipula cuál es la versión que de esa historia se propone. Esa versión pone a los investigadores, profesores de ciencias y estudiantado ante una concepción y, por tanto, frente a una aproximación que puede ser o no tergiversada o que recoge o no la complejidad de los procesos mediante los cuales fue necesario proponer teorías o modelos científicos (GRECA e DOS SANTOS, 2005) a las problemáticas de cada tiempo, como también del papel cumplido por los correspondientes colectivos de especialistas ese proceso histórico.

En el planteamiento de esta relación es indispensable la ubicación temporal, con el fin de explicitar a qué concepción de ciencia se alude (ECHEVERRÍA, 2003), dentro de la explicable intencionalidad de que los didactas se ocupan de socializar unas teorías o modelos, cuyo significado tendría connotación de ser histórico. Este es uno de los problemas que establece una relación entre con la epistemología y la didáctica, sobre todo si de aeduacón científica en los niveles básico y secundario se trata.

La relación historia $(\mathrm{H})$ y didáctica de las ciencias $(\mathrm{D})$ existirá si en un sistema educativo dado, la historia se considera indispensable para una mejor comprensión entre el estudiantado de esas razones por las cuales se propusieron las teorías o modelos científicos que se hacen objeto de trabajo en el aula (IZQUIERDO, 1988). Será necesaria si los programas académicos de formación inicial y continua de profesores de ciencias han tenido esa historia como uno de los ejes de dicha formación (GALLEGO BADILLO e GALLEGO TORRES, 2003). Se trata, por supuesto, de un tratamiento tanto externo como interno de esa historia, referida a cada teoría o modelo.

La relación epistemología (E) y didáctica (D), en primer lugar, precisa a qué didáctica se hace referencia y se pone en práctica en el colectivo aula. Desde la aproximación empiropositivista y tecnicista, esa didáctica tendrá un carácter meramente instrumental, esto es, reducida a unos métodos de enseñanza, en el contexto habitual en la que es asumida como la parte operativa de la pedagogía, en la que esta última es considerada, sin mejores análisis epistemológicos, como la ciencia de la educación. Si la aproximación es deductivista-constructivista, la 
Historia, epistemología y didáctica...

didáctica será asumida como una disciplina conceptual y metodológicamente fundamentada (GALLEGO TORRES e GALLEGO BADILLO, 2005), en la que dos de los problemas centrales son los de la enseñabilidad y la enseñanza. El primero, que se resuelve desde la transposición (CHEVALLARD, 1985), a partir de unos supuestos didácticos que han de ser sometidos a contrastación sistemática en el colectivo aula, mediante la formulación de unas actividades didácticas que son la solución al segundo.

Hay otras relaciones, indicadas por las dobles flechas internas, que indican la complejidad que recoge el triángulo que aquí se propone. En efecto, la aproximación epistemológica (E) que se asuma, afecta la relación entre historia $(\mathrm{H})$ y didáctica (D), ya señalada. Esto es, la teoría o modelo científico que se hace objeto de trabajo en el aula estará marcada por esa opción y, consecuentemente, determinará el tipo de contenido curricular y la clase de trabajo que adelantará el profesor de ciencias con sus estudiantes. La concepción que de la didáctica (D) de las ciencias se haya elaborado, reclamará una y será solidaria con una clase de mirada epistemológica $(\mathrm{E})$ y con la histórica $(\mathrm{H})$. Ello significa que establecida una posición didáctica, esta no se aviene con cualquier posición epistemológica y, de esta manera, con cualquier versión histórica. Esta relación es de tener en cuenta, por cuanto de aceptarse, deja sentado que la didáctica (D) se encuentra en el mismo plano de importancia que tienen la historia y la epistemología (ADÚRIZ-BRAVO e IZQUIERDO, 2002; GALLEGO BADILLO, 2004).

La versión histórica (H) que se adopte (SERRES, 1991), incidirá en las relaciones entre epistemología (E) y didáctica (D), por las razones que ya se han aludido en este artículo, sobre las cuales no habría que insistir. No obstante, hay que señalar que los cambios en las aproximaciones epistemológicas $(\mathrm{E})$, en las versiones acerca de la historia $(\mathrm{H})$ y en las concepciones en torno a la didáctica de las ciencias (D), generan paulatinamente perturbaciones en las relaciones sobre las cuales internamente inciden, las que a largo plazo, a su vez, modifican dichas relaciones.

\section{Unas conclusiones para tener en cuenta}

Las propuestas epistemológicas de Kuhn (1972) y de Lakatos (1983), pusieron de presente la necesidad de apoyarse en una revisión de la historia del desarrollo de las teorías o modelos científicos, como condición para fundamentar una explicación epistemológica acerca del carácter del conocer y del conocimiento en las ciencias de la naturaleza. Los dos, llamados epistemólogos historicistas, enseñaron que no era justificable una y sólo una historia de las ciencias, ya que todo dependía del punto de vista epistemológico a partir del cual esa historia cobraba sentido.

Las elaboraciones de los autores citados, dieron pie para introducir en la nueva didáctica de las ciencias el problema de la historia interna y externa de las teorías y modelos científicos que se hacen objeto de trabajo en el aula, a la vez que examinar, con respecto a cada sistema educativo, la versión epistemológica y didáctica a partir de la cual ciertas versiones acerca de esas teorías o modelos, por ley, se hacen contenido curricular.

¿Cómo elaborar propuestas de educación en ciencias en las que sea posible un enfoque integral como el que recoge la triangulación aquí presentada? ¿Hasta donde ir más allá de una mera utilización de la historia de las ciencias y de cada teoría o modelo científico como 
Gallego Torres, A. P.; Gallego Badillo, R.

simple anecdotario? ¿Qué características ha de delimitar un proyecto de investigación didáctica que tome en consideración la triangulación propuesta como fundamento conceptual y metodológico? ¿De qué manera desarrollar en profundidad, con ejemplos específicos, las relaciones que se proponen?

\section{Referências}

ACEVEDO, J. A.; VÁSQUEZ, A.; PAIXÃO, M. F.; et al. Mitos da didática das ciências acerca dos motivos para incluir a natureza da ciência no ensino das ciências. Ciência $\boldsymbol{\&}$ Educação, Bauru, v. 11, n. 1, p. 1-16, 2005.

ADÚRIZ-BRAVO, A.; IZQUIERDO, M. Acerca de la didáctica de las ciencias como disciplina autónoma. Revista Electrónica de Enseñanza de las Ciencias, Vigo, v. 1, n. 3, artigo 1, 2002. Disponível em: <http://www.saum.uvigo.es/reec>.

BRIGGS, J.; PEAT, F. D. Espejo y reflejo: del caos al orden. Barcelona: Gedisa, 1990.

CALDIN, E. F. The structure of chemistry in relation to the philosophy of science.

International Journal for Philosophy of Chemistry, Alemanhã, v. 8, n. 2, p.103-121, 2002. Disponível em: <http://.hyle.org/journal/issues/8-2/caldin.html>.

CHEVALLARD, Y. La transposition didactique: du savoir savant au savoir enseigné. Grenoble: La Pensée Sauvage, 1985.

DEL RE, G. Models and analogies in science. International Journal for Philosophy of Chemistry, Alemanha, v. 6, n. 1, p. 5-15, 2000. Disponível em: <http://www.hyle.org/ journal/issues/6/delre.html>.

ECHEVERRÍA, J. La revolución tecnocientífica. Madrid: FCE, 2003.

FEYERABEND, P. K. Contra el método: esquema de una teoría anarquista del conocimiento. Barcelona: Orbis, 1984.

GALLEGO BADILLO, R. Un concepto epistemológico de modelo para la didáctica de las ciencias experimentales. Revista Electrónica de Enseñanza de las Ciencias, Alemanha, v. 3, n. 4, artigo 4, 2004. Disponível em: <http://www.saum.uvigo.es/reec>.

.; GALLEGO TORRES, A. P. La formación inicial de profesores de ciencias: un problema didáctico y curricular. Tecné, Episteme y Didaxis, Bogotá, número extra, p. 66$74,2003$.

R.; et al. La formación inicial de profesores de ciencias en Colombia: contrastación de fundamentos. Bogotá: Universidad Pedagógica Nacional, 2004. (Memoria de investigación). 
Historia, epistemología y didáctica...

.; PÉREZ MIRANDA, R.; TORRES DE GALLEGO, L. N. Formación inicial de profesores de ciencias en Colombia: un estudio a partir de programas acreditados. Ciência \& Educação, Bauru, v. 10, n. 2, p. 219-234, 2004.

.; et al. El concepto de valencia: su construcción histórica y epistemológica y la importancia de su inclusión en la enseñanza. Ciência $\boldsymbol{\&}$ Educação, Bauru, v. 10, n. 3, p. 571-583, 2004.

GALLEGO TORRES, A. P. Contribución del cómic a la imagen de la ciencia. Valencia, 2002. Tesis (Doctoral) - Universidad de Valencia.

.; GALLEGO BADILLO, R. El estatuto científico de la nueva didáctica de las ciencias. (Ponencia). Tecné, Episteme y Didaxis, Bogotá, número extra, p. 221-223, 2005.

GIERÉ, R. N. Explaining science. Chicago: University of Chicago Press, 1990.

GRECA, I. M.; DOS SANTOS, F. M. T. Dificuldades da generalização das estratégias de modelação em ciências: o caso da física e da química. Investigações em Ensino de Ciências, 2005. Disponível em: <http://www.if.ufrgs.br/public/ensino/vol10/n1/ v10_n1_a2.htm>. Acesso em: 20 maio 2004.

HANSON, N. R. Observation and explanation: a guide to philosophy of science. Patterns of discovery: an inquiry into the conceptual foundations of science. Cambridge: Cambridge University Press, 1977.

HODSON, D. Philosophy of science, science and science education. Studies in Science Education, Barcelona, v. 12, n. 1, p. 25-27, 1985.

HOFFMANN, R. Lo mismo y no lo mismo. México: Fondo de Cultura Económica, 1997.

IZQUIERDO, M. La contribución de la teoría del flogisto a la estructuración actual de la ciencia química. Implicaciones didácticas. Enseñanza de las Ciencias, Barcelona, v. 6, n. 1, p. 67-74, 1988.

KRETZENBACHER, H. L. The aesthetics and Heuristics of Analogy. Model and Metaphor in Chemical Communication. International Journal for Philosophy of Chemistry, Alemanha, v. 9, n. 2, p.199-218, 2003. Disponível em: < http://www.hyle.org/ journal/issues/9-2/kretzenbacher.html>.

KUHN, T. S. La estructura de las revoluciones científicas. México: Fondo de Cultura Económica, 1972.

LEIDLER, K. J. The world of physical chemistry. Oxford: Oxford University Press, 1995.

LAKATOS, I. La metodología de los programas de investigación científica. Madrid: Alianza, 1983. 
Gallego Torres, A. P.; Gallego Badillo, R.

LOMBARDI, O. La noción de modelo en ciencias. Educación en Ciencias, Buenos Aires, v. 2, n. 4, p. 5-13, 1998.

MATTEWS, M. R. In defense of modest goals when teaching about the nature of science. Journal of Research in Science Teaching, New York, v. 35, n. 2, p. 161-174, 1998.

POPPER, K. La lógica de la investigaxción cientíifica. Madrid: Tecnos, 1962.

PORLÁN, R. Teoría del conocimiento, teoría de la enseñanza y desarrollo profesional: las concepciones epistemológicas de los profesores. Sevilla, 1989. Tesis (Doctoral) - Universidad de Sevilla.

RUELLE, D. Azar y caos. Madrid: Alianza, 1993.

SCHELER, M. Erkenntnis und arbeit. Leipzig: Der neue Geist,1926.

SERRES, M. Prefacio que invita al lector a no descuidar su lectura para penetrar en la intención de los autores y comprender la disposición de este libro. In: Historia de las ciencias. Madrid: Cátedra, 1991. p. 9-25.

TOMASI, J. Towards "chemical congruence" of the models in theoretical chemistry. International Journal for Philosophy of Chemistry, Alemanha, v. 5, n. 2, p. 79-115, 1999. Disponible: <http://.hyle.org/journal/issues/5/tomasi.html>.

TOULMIN, S. La comprensión humana: el uso colectivo y la evolución de los conceptos. Madrid: Alianza, 1977. v. 1.

ZAHAR, E. Experimentos cruciales: estudio de un ejemplo. In: RADNITZKY, G.; ANDERSSON, G. (Eds.). Progreso y racionalidad en la ciencia. Madrid: Alianza, 1980. p. 70-94.

Artigo recebido em julho de 2006 e aceito em fevereiro de 2007. 